\title{
The XRCC1 Arg194Trp polymorphism is not a risk factor for glioma: A meta-analysis involving 1,440 cases and 2,562 controls
}

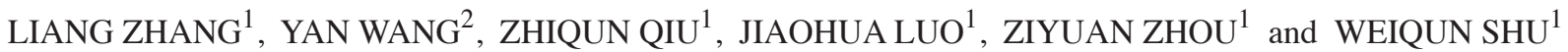 \\ ${ }^{1}$ Department of Environmental Hygiene, College of Preventive Medicine, \\ Third Military Medical University, Chongqing 400038; ${ }^{2}$ Institute of Respiratory Diseases, \\ Xinqiao Hospital, Third Military Medical University, Chongqing 400037, P.R. China
}

Received July 5, 2012; Accepted September 13, 2012

DOI: $10.3892 /$ etm.2012.716

\begin{abstract}
Previous reports have indicated that the X-ray repair cross-complementing gene 1 (XRCC1) Arg194Trp polymorphism may be a risk factor for several types of cancer. Published studies on the association of XRCC1 Arg194Trp polymorphisms with glioma risk have yeilded conflicting results. The present study aimed to obtain a more precise estimation of this association. Meta-analyses assessing the association of the XRCC1 Arg194Trp variation with glioma were conducted and subgroup analyses based on ethnicity and source of controls were also performed. Eligible studies were identified during the period before May 2012. A total of four case-control studies comprising 1,440 cases and 2,562 controls were finally selected for analysis. The overall data failed to indicate a significant association of the XRCC1 Arg194Trp polymorphism with glioma risk [Trp vs. Arg: odds ratio $(\mathrm{OR})=1.01,95 \%$ confidence interval $(95 \% \mathrm{CI})=0.77-1.33$; Trp/Trp vs. Arg/Arg: OR = 1.56, 95\% CI = 0.96-2.54; dominant model: $\mathrm{OR}=0.98,95 \% \mathrm{CI}=0.74-1.31$; recessive model: $\mathrm{OR}=1.48,95 \% \mathrm{CI}=0.92-2.38]$. Similarly, in the subgroup analysis based on ethnicity and source of controls, no associations were observed. In conclusion, the results of the present study failed to suggest an association between the XRCC1 Arg194Trp polymorphism and glioma risk. Further large and well-designed studies are required to confirm this conclusion.
\end{abstract}

\section{Introduction}

Glioma is the most common type of primary brain tumor in adults. The general outcomes for patients are poor, particularly

Correspondence to: Professor Weiqun Shu, Department of Environmental Hygiene, College of Preventive Medicine, Third Military Medical University, 30 Gaotanyan Street, Chongqing 400038, P.R. China

Email: sydswq@163.com

Key words: X-ray repair cross-complementing gene 1 Arg194Trp, glioma, malignancy, susceptibility, meta-analysis, polymorphism for older patients. The etiology of glioma is unclear. Evidence suggests that exposure to radiation may be a significant risk factor for glioma, which may explain a small proportion of glioma since this exposure is generally rare (1). However, only a minority of individuals exposed to radiation eventually develop glioma, indicating that host genetic factors may play a critical role in the carcinogenesis of glioma (2).

Radiation exposure may cause DNA damage and cell injury. The consequences to the cells may be disastrous, ranging from single gene mutations to massive chromosomal breakdown and rearrangements. The instabilities may result in severe human disorders, including cancer (3). The repair of various types of DNA damage is vital for the maintenance of genomic stability and cell survival. Base excision repair pathways are critical in this process. X-ray repair cross-complementing gene 1 (XRCC1) is one of the most important DNA repair genes that plays a key role in the process of base excision repair. The XRCC1 gene is located on chromosome 19q13.2-13.3 and is $33 \mathrm{~kb}$ in length, comprising 17 exons and encoding a 70-kDa protein (4). A widely studied XRCC1 single nucleotide polymorphism at codon 194, with a Arg to Trp alteration (rs1799782), may have a diminished capacity to remove DNA adducts and oxidized DNA damage (5). Hence, the Arg194Trp variation has been associated with cancer susceptibility.

Published data on the association of the XRCC1 Arg194Trp polymorphism with glioma have yielded controversial results. In the present study, we carried out a quantitative meta-analysis that increased statistical power to derive a more precise estimation of this association.

\section{Materials and methods}

Literature search strategy. We performed a search of the Medline, Embase, Ovid, Sciencedirect and Chinese National Knowledge Infrastructure (CNKI) databases without a language limitation, including all studies published until May 2012, with a combination of the following keywords: XRCC1, Arg194Trp, glioma, brain, neoplasm, cancer, variation and polymorphism. All searched studies were retrieved and the bibliographies were reviewed for other relevant publications. Review articles and bibliographies of other relevant studies identified were searched manually to identify additional eligible studies. 
Inclusion criteria. The following criteria were used for the literature selection: i) studies should report the association of the XRCC1 Arg194Trp polymorphism with glioma risk; ii) studies are observational studies (case-control or cohort); iii) studies should provide the sample size, odds ratios (ORs) and $95 \%$ confidence intervals (CIs), the genetic distribution or the information to infer the results. After rigorous searching, we reviewed all studies in accordance with the criteria defined above for further analysis.

Data extraction. Data were carefully extracted from all eligible publications independently by two of the authors according to the inclusion criteria mentioned above. For conflicting evaluations, an agreement was reached following a discussion. If a consensus could not be reached, another author was consulted to resolve the dispute and then a final decision was made by a majority of the votes. Extracted information was entered into a database.

Statistical analysis. The OR of the XRCC1 Arg194Trp polymorphism and glioma risk was estimated for each study. The pooled ORs were assessed for the genetic comparisons of allelic contrast (Trp vs. Arg), homozygote comparison (Trp/Trp vs. Arg/Arg), dominant model (Trp/Trp + Trp/Arg vs. Arg/Arg) and recessive model (Trp/Trp vs. Trp/Arg + Arg/Arg). For the detection of any possible sample size biases, the OR and 95\% CI for each study was plotted against the number of participants. A Chi-square based Q statistic test was performed to assess heterogeneity. If the result of the heterogeneity test was $\mathrm{P}>0.1$, ORs were pooled according to the fixed-effects model (Mantel-Haenszel); otherwise, the random-effects model (DerSimonian and Laird) was used. The significance of the pooled ORs was determined by the Z-test. The Hardy-Weinberg equilibrium (HWE) was assessed by Fisher's exact test.

Publication bias was assessed by visual inspection of funnel plots (6), in which the standard error of $\log (\mathrm{OR})$ of each study was plotted against its $\log (\mathrm{OR})$. An asymmetric plot indicates a possible publication bias. The symmetry of the funnel plot was further evaluated by Egger's linear regression test (7). Statistical analysis was undertaken using the program STATA 11.0 software (Stata Corporation, College Station, TX, USA).

\section{Results}

Study characteristics. Relevant publications were retrieved and screened. As shown in Fig. 1, a total of 42 publications were identified, of which 34 irrelevant studies were excluded. Thus, eight publications were eligible in preliminary stages, of which one review article (8) and one non-case-control study (9) were discarded. Subsequently, one study that did not provide the detailed distributions of the genotypes (10) was excluded. Five case-control studies were included for data extraction and analysis (11-15). However, a study conducted by Custodio et al (13) was excluded since it contributed to evident heterogeneity for the overall data under the four genetic models. Lasty, four case-control studies were selected for analysis $(11,12,14,15)$.

All the selected publications were written in English. The relevant information is listed in Table I, including the first author and the number and characteristics of cases and controls for each study as well as other relevant information. There were

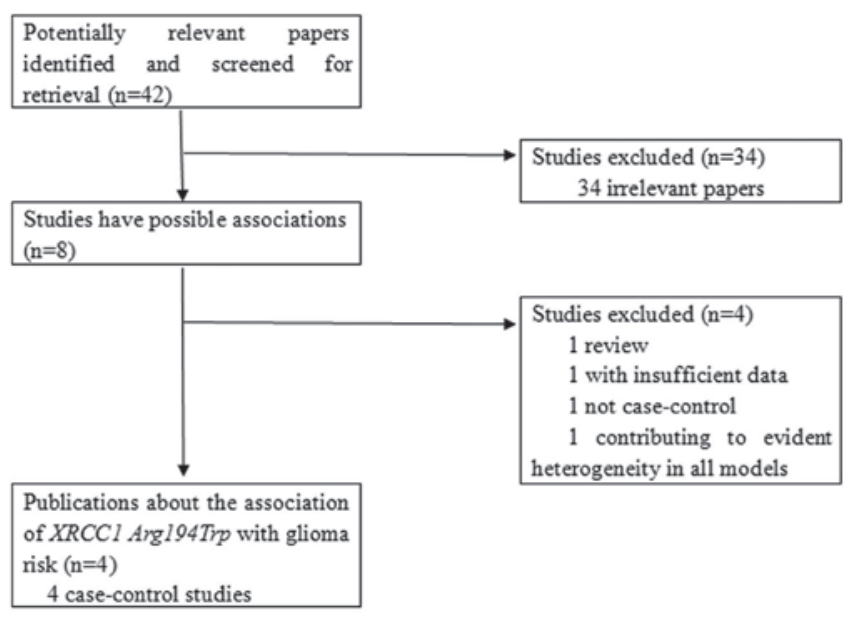

Figure 1. Flow diagram of included and excluded studies.

two groups of Asians $(14,15)$ and two of Caucasians $(11,12)$ in the present meta-analysis.

The distributions of the XRCC1 Arg194Trp genotypes as well as the genotyping methods of the included studies are presented in Table II. The genetic distributions of the control groups in all studies were consistent with the HWE, with the exception of one study (14).

Test of heterogeneity. As shown in Table III, we analyzed the heterogeneities for the four genetic comparisons. Evident heterogeneities for the overall data were present in the allelic contrast $(\mathrm{P}=0.030, \mathrm{Q}$-test) and dominant model $(\mathrm{P}=0.061$ for $\mathrm{Q}$-test), with the exception of the homozygote comparison $(\mathrm{P}=0.561, \mathrm{Q}$-test $)$ and recessive model $(\mathrm{P}=0.598, \mathrm{Q}$-test $)$. Additionally, the I-square value is another index for a heterogeneity test (16), with value less than $25 \%$ indicating low, $25 \%$ to $50 \%$ indicating moderate, and greater than $50 \%$ indicating high heterogeneity. The I-square values were 66.6 and $59.2 \%$ for the overall data of the allelic contrast and dominant model, respectively, suggesting statistically significant heterogeneity between the studies; therefore, the random-effects models were utilized. For the homozygote comparison and recessive model, the I-square values were both $0.0 \%$, indicating an absence of the heterogeneity. Thus, the fixed-effects model was used in these two models.

Notably, when the overall data were divided for subgroup analysis, we observed a loss of heterogeneity in the subgroups in the allelic contrast and dominant models.

Meta-analysis results. The main results of the meta-analysis are listed in Table III. For the overall data including 1,440 cases and 2,562 controls, significant associations of the XRCC1 Arg194Trp polymorphism with glioma risk were not identified in the four genetic models (allele contrast: $\mathrm{OR}=1.01,95 \% \mathrm{CI}=0.77-1.33$; homozygote comparison: $\mathrm{OR}=1.56,95 \% \mathrm{CI}=0.96-2.54$; dominant model: $\mathrm{OR}=0.98,95 \% \mathrm{CI}=0.74-1.31$; recessive model: $\mathrm{OR}=1.48,95 \% \mathrm{CI}=0.92-2.38)$, indicating that the XRCC1 Arg194Trp polymorphism may not have a correlation with glioma risk.

Considering the possible effects of ethnic variation and selection of controls on the results (Fig. 2), we conducted 


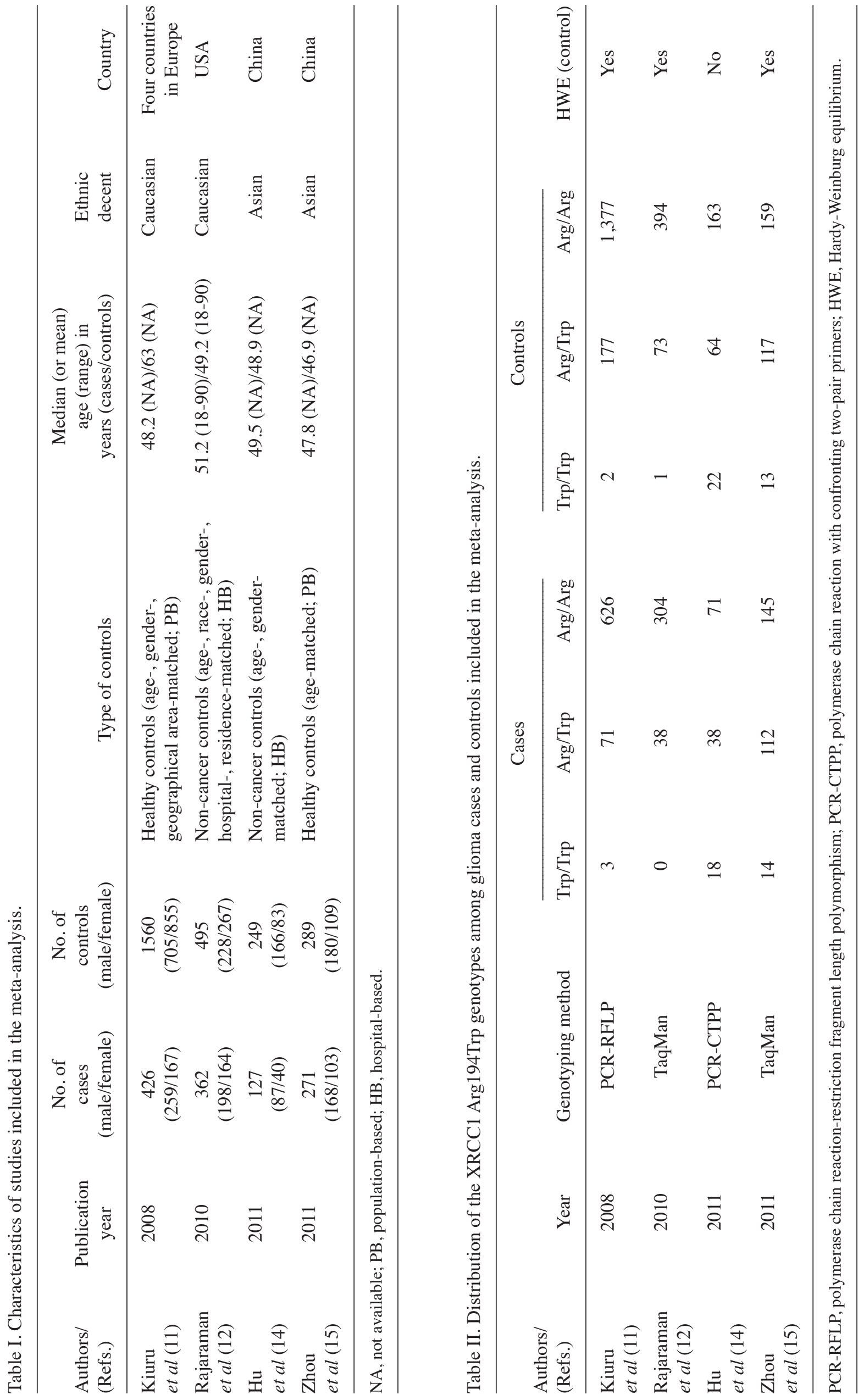



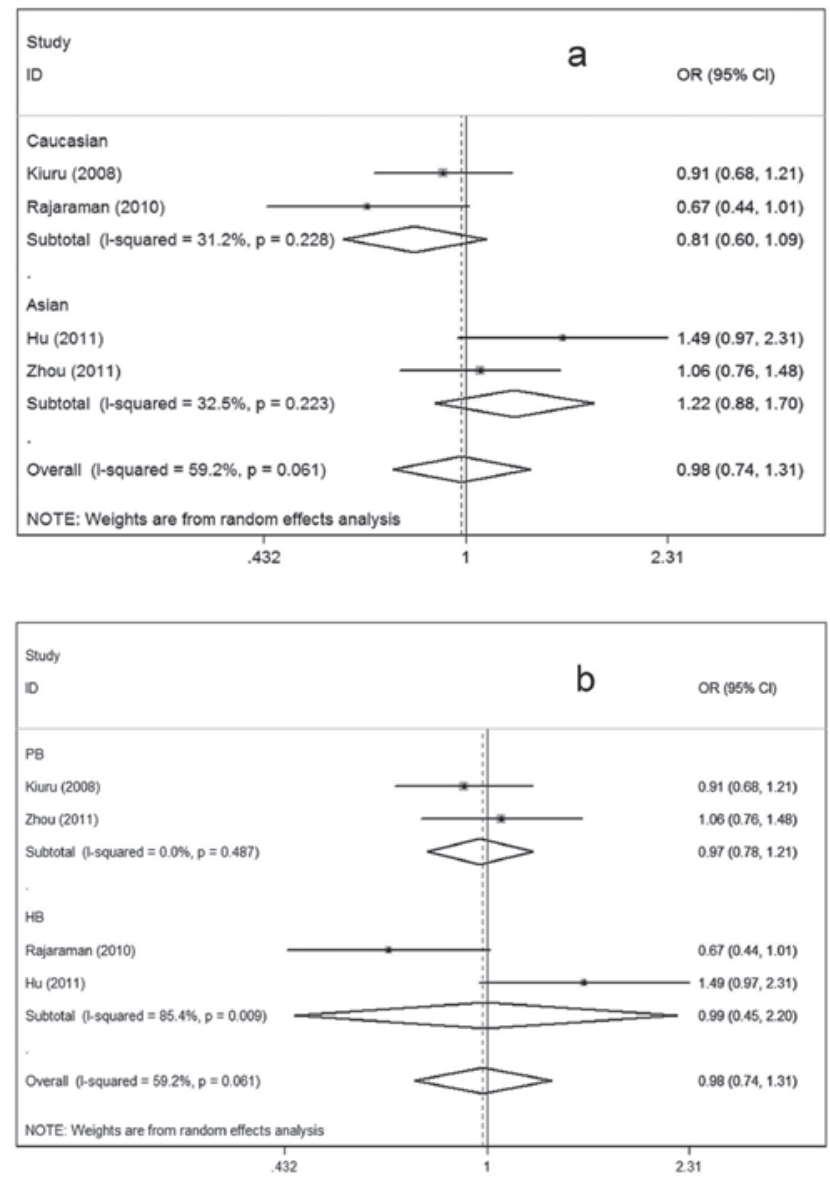

Figure 2. Meta-analysis for the association of glioma risk with the X-ray repair cross-complementing gene 1 (XRCC1) Arg194Trp polymorphism. (Trp/Trp + Trp/Arg vs. Arg/Arg). (A) Stratified by ethnicity. (B) Stratified by source of controls. OR, odds ratio; $\mathrm{CI}$, confidence interval.

subgroup analyses. In the subgroup analysis according to ethnicity, no associations were observed in either the Asian or Caucasian subgroup (Fig. 2a). Similarly, in the subgroup analysis by source of controls, significant associations could be observed in neither the population-based subgroups nor the hospital-based subgroups under the four genetic comparisons (Fig. 2b).

Sensitivity analysis. To test the stability of the overall results, we carried out one-way sensitivity analysis (17). The statistical significance of the results was not changed when any single study was omitted (data not shown), indicating the robustness of the results.

Bias diagnostics. Funnel plots were created for assessment of possible publication biases (Fig. 3a). Then, Egger's linear regression tests were used to assess the symmetries of the plots. The funnel plots appeared to be symmetrical for the overall data indicated by the Egger's tests (allelic contrast: $\mathrm{t}=-0.33, \mathrm{P}>0.05$, homozygote comparison: $\mathrm{t}=-0.24, \mathrm{P}>0.05$; 

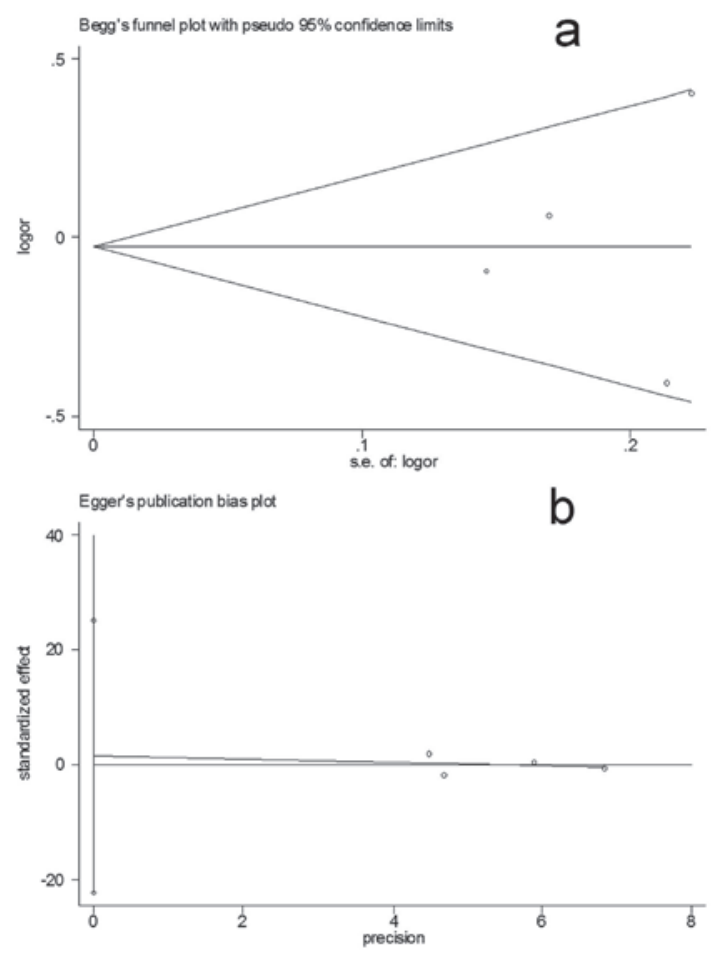

Figure 3. Publication bias test for the overall data (Trp/Trp + Trp/Arg vs. Arg/Arg). (A) Funnel plot. (B) Egger's linear regression test.

dominant model: $\mathrm{t}=0.26, \mathrm{P}>0.05$; recessive model: $\mathrm{t}=-0.13$, $\mathrm{P}>0.05$; Fig. $3 \mathrm{~b}$ ), suggesting that the publication biases were not evident.

\section{Discussion}

The overall data obtained failed to show a significant association between the XRCC1 Arg194Trp polymorphism and glioma risk. Similar results were demonstrated in the subgroup analysis based on ethnicity and source of controls.

Previous meta-analyses have focused on the association between the XRCC1 Arg194Trp polymorphism and several cancer risks and have generated conflicting results. The XRCC1 Arg194Trp variation has been demonstrated to increase risk of lung and gastric cancer $(18,19)$. However, meta-analyses regarding skin and esophageal cancer failed to reveal such associations $(20,21)$. Therefore, the XRCC1 Arg194Trp polymorphism may exert different effects on different cancers.

In the subgroup analysis based on ethnicity, no significant associations could be observed in the Asian or Caucasian subgroup. Evidence indicates that ethnic-specific variation, different health care and socioeconomic class might exert an effect on the incidence of glioma (22). The results demonstrated that the potential effects of ethnic variations on glioma were not evident. Notably, the results should be interpreted with care since the limited number of the included studies containing small sample sizes may result in insufficient statistical power to evaluate a small effect. Therefore, future investigations regarding different ethnicities with large sample sizes are required to clarify this issue.

When the data were stratified based on the source of controls, no association was observed in either the population-based or hospital-based subgroup. Since hospital-based controls may not be representative of the general population, a selection bias may exist. However, the data of the present meta-analysis suggest that the potential influence of the selection bias on the overall results was not evident. However, use of proper control participants with rigorous matching criteria and large sample sizes in future studies is important to reduce such possible selection bias.

In the present meta-analysis, evident between-study heterogeneities for overall data were observed in the allelic contrast and dominant models. However, when the subgroup analyses were conducted, we found that the heterogeneities were removed in the subgroups regarding Caucasian, Asian and population-based controls. However, the heterogeneity was still present in the hospital-based subgroup. Therefore, the heterogeneities might be multifactorial. In addition to ethnicity and source of controls, other factors including age, pathology grade and life styles may also contribute to the heterogeneity.

Several limitations should be addressed. First, in this meta-analysis, the primary articles only provided data regarding Caucasians, Asians and mixed races. Other ethnicities such as African should be investigated in future studies. Second, subgroup analyses based on age, gender, histological types, radiation exposure and other factors have not been performed in the present study since sufficient relevant data were not available in the primary literature. Third, only studies written in English and several other languages indexed by the common databases were searched. Thus, a selection bias might exist. Therefore, the results should be interpreted with caution. However, the sensitivity analysis and publication bias analysis indicated the stability and credibility of the present meta-analysis.

In conclusion, the results of the present meta-analysis failed to suggest an association of the XRCC1 Arg194Trp polymorphism with glioma risk. Further investigations with larger sample sizes and rigorous matching criteria in view of confounding factors are required to confirm the associations.

\section{References}

1. Prasad G and Haas-Kogan DA: Radiation-induced gliomas. Expert Rev Neurother 9: 1511-1517, 2009.

2. Melin B: Genetic causes of glioma: new leads in the labyrinth. Curr Opin Oncol 23: 643-647, 2011.

3. Li GM: A special issue on DNA damage response and genome stability. Cell Biosci 2: 4, 2012.

4. Mutamba JT, Svilar D, Prasongtanakij S, et al: XRCC1 and base excision repair balance in response to nitric oxide. DNA Repair (Amst) 10: 1282-1293, 2011.

5. Ginsberg G, Angle K, Guyton K and Sonawane B: Polymorphism in the DNA repair enzyme XRCC1: utility of current database and implications for human health risk assessment. Mutat Res 727: 1-15, 2011.

6. Munafo MR, Clark TG and Flint J: Assessing publication bias in genetic association studies: evidence from a recent meta-analysis. Psychiatry Res 129: 39-44, 2004.

7. Egger M, Davey Smith G, Schneider M and Minder C: Bias in meta-analysis detected by a simple, graphical test. BMJ 315: 629-634, 1997.

8. Goode EL, Ulrich CM and Potter JD: Polymorphisms in DNA repair genes and associations with cancer risk. Cancer Epidemiol Biomarkers Prev 11: 1513-1530, 2002. 
9. Liu Y, Shete S, Hosking F, Robertson L, Houlston R and Bondy M: Genetic advances in glioma: susceptibility genes and networks. Curr Opin Genet Dev 20: 239-244, 2010.

10. Liu Y, Scheurer ME, El-Zein R, et al: Association and interactions between DNA repair gene polymorphisms and adult glioma. Cancer Epidemiol Biomarkers Prev 18: 204-214, 2009.

11. Kiuru A, Lindholm C, Heinavaara S, et al: XRCC1 and XRCC3 variants and risk of glioma and meningioma. J Neurooncol 88: $135-142,2008$

12. Rajaraman $\mathrm{P}$, Hutchinson $\mathrm{A}$, Wichner $\mathrm{S}$, et al: DNA repair gene polymorphisms and risk of adult meningioma, glioma, and acoustic neuroma. Neuro Oncol 12: 37-48, 2010.

13. Custodio AC, Almeida LO, Pinto GR, et al: Analysis of the polymorphisms XRCC1Arg194Trp and XRCC1Arg399Gln in gliomas. Genet Mol Res 10: 1120-1129, 2011.

14. Hu XB, Feng Z,Fan YC, Xiong ZY and Huang QW: Polymorphisms in DNA repair gene $\mathrm{XRCC} 1$ and increased genetic susceptibility to glioma. Asian Pac J Cancer Prev 12: 2981-2984, 2011.

15. Zhou LQ, Ma Z, Shi XF, et al: Polymorphisms of DNA repair gene XRCC1 and risk of glioma: a case-control study in Southern China. Asian Pac J Cancer Prev 12: 2547-2550, 2011.
16. Higgins JP, Thompson SG, Deeks JJ and Altman DG: Measuring inconsistency in meta-analyses. BMJ 327: 557-560, 2003.

17. Tobias A: Assessing the influence of a single study in the meta-analysis estimate. Stata Techn Bull 8: 15-17, 1999.

18. Cui Z, Yin Z, Li X, Wu W, Guan P and Zhou B: Association between polymorphisms in XRCC1 gene and clinical outcomes of patients with lung cancer: a meta-analysis. BMC Cancer 12: 71, 2012.

19. Chen B, Zhou Y, Yang P and Wu XT: Polymorphisms of XRCC1 and gastric cancer susceptibility: a meta-analysis. Mol Biol Rep 39: 1305-1313, 2012.

20. Zhang H, Li W, Franklin MJ and Dudek AZ: Polymorphisms in DNA repair gene XRCC1 and skin cancer risk: a meta-analysis. Anticancer Res 31: 3945-3952, 2011.

21. Yin M, Tan D and Wei Q: Genetic variants of the XRCC1 gene and susceptibility to esophageal cancer: a meta-analysis. Int J Clin Exp Med 2: 26-35, 2009 .

22. Curry WT Jr and Barker FG II: Racial, ethnic and socioeconomic disparities in the treatment of brain tumors. J Neurooncol 93: 25-39, 2009. 